\title{
CMD-3 Overview
}

Ivan B. Logashenko ${ }^{1,2, \star, \star \star}$ F.V.Ignatov ${ }^{1}$, R.R.Akhmetshin ${ }^{1}$, A.N.Amirkhanov ${ }^{1,2}$, A.V.Anisenkov ${ }^{1,2}$, V.M.Aulchenko, ${ }^{1,2}$, V.S.Banzarov ${ }^{1}$, N.S.Bashtovoy ${ }^{1}$, D.E.Berkaev ${ }^{1,2}$, A.E.Bondar ${ }^{1,2}$, A.V.Bragin ${ }^{1}$, S.I.Eidelman ${ }^{1,2}$, D.A.Epifanov ${ }^{1,2}$, L.B.Epshteyn ${ }^{1,2,3}$, A.L.Erofeev ${ }^{1,2}$, G.V.Fedotovich ${ }^{1,2}$, S.E.Gayazov ${ }^{1,2}$, A.A.Grebenuk ${ }^{1,2}$, S.S.Gribanov ${ }^{1,2}$, D.N.Grigoriev ${ }^{1,2,3}$, V.L.Ivanov ${ }^{1,2}$, S.V.Karpov ${ }^{1}$, V.F.Kazanin 1,2, A.N.Kirpotin ${ }^{1}$, A.A.Korobov ${ }^{1,2}$, I.A.Koop ${ }^{1}$, A.N.Kozyrev 1,2, E.A.Kozyrev ${ }^{1,2}$, P.P.Krokovny ${ }^{1,2}$, A.E.Kuzmenko ${ }^{1,2}$, A.S.Kuzmin ${ }^{1,2}$, P.A.Lukin ${ }^{1,2}$, K.Yu.Mikhailov ${ }^{1,2}$, V.S.Okhapkin ${ }^{1}$, A.V.Otboev ${ }^{1}$, Yu.N.Pestov ${ }^{1}$, A.S.Popov ${ }^{1,2}$, G.P.Razuvaev ${ }^{1,2}$, A.A.Ruban ${ }^{1}$, N.M.Ryskulov ${ }^{1}$, A.E.Ryzhenenkov ${ }^{1,2}$, A.I.Senchenko ${ }^{1,2}$, V.E.Shebalin ${ }^{1,2}$, D.N.Shemyakin ${ }^{1,2}$, B.A.Shwartz ${ }^{1,2}$, D.B.Shwartz ${ }^{1,2}$, A.L.Sibidanov ${ }^{4}$, P.Yu.Shatunov ${ }^{1}$, Yu.M.Shatunov ${ }^{1,2}$, E.P.Solodov ${ }^{1,2}$, A.A.Talyshev ${ }^{1,2}$, A.I.Vorobiov ${ }^{1}$, Yu.V.Yudin ${ }^{1,2}$, I.M.Zemlyansky ${ }^{1}$

${ }^{1}$ Budker Institute of Nuclear Physics, SB RAS, Novosibirsk, 630090, Russia

${ }^{2}$ Novosibirsk State University, Novosibirsk, 630090, Russia

${ }^{3}$ Novosibirsk State Technical University, Novosibirsk, 630092, Russia

${ }^{4}$ University of Victoria, Victoria, British Columbia, Canada V8W 3P6

\begin{abstract}
The CMD-3 detector is installed at the VEPP-2000 $e^{+} e^{-}$collider at BINP (Novosibirsk, Russia). It is a general-purpose detector, equipped with a tracking system, two crystal (CSI and BGO) calorimeters, liquid Xe calorimeter, TOF and muon systems. The main goal of experiments at CMD-3 is a study of exclusive modes of $e^{+} e^{-} \rightarrow$ hadrons at energies $\sqrt{s} \leq 2 \mathrm{GeV}$. In particular, these results provide an important input for calculation of the hadronic contribution to the muon anomalous magnetic moment.

The first round of data taking was performed in 2011-2013, when about $601 / \mathrm{pb}$ were taken in the center-of-mass (c.m.) energy range from 0.32 to $2.0 \mathrm{GeV}$. Here we present a survey of results of data analysis.

Between 2013 and 2016 the collider and the detector were upgraded. The data taking resumed by the end of 2016. In the first run after the upgrade about $501 / \mathrm{pb}$ were collected at the energy range between 1.28 and $2.007 \mathrm{GeV}$. We discuss the upgrade and the first preliminary results from the new data.
\end{abstract}

\section{Introduction}

The electron-positron collider VEPP-2000[1, 2] started operation at Budker Institute of Nuclear Physics (Novosibirsk, Russia) in 2010. The machine covers the c.m. energy range from $\sqrt{s}=0.32$ $\mathrm{GeV}$ to $2.0 \mathrm{GeV}$ and employs the technique of round beams. Two detectors, CMD-3[3] and SND[4] are installed in the two interaction regions of VEPP-2000.

\footnotetext{
^e-mail: I.B.Logashenko@inp.nsk.su

$\star \star$ This work is supported in part by the Russian Science Foundation (project 14-50-00580) and RFBR grants 16-02-00160-a, 17-02-00327-a, 17-02-00847-a, 17-02-00897-a.
} 
The CMD-3 (Fig. 1) is a general-purpose particle detector. The cylindrical drift chamber with hexagonal cells is surrounded by the Z-chamber, a MWPC with a dual anode and cathode readout, used for precise determination of the fiducial volume for charged particles. The barrel electromagnetic calorimeter [5], placed outside the superconducting solenoid $\left(0.13 \mathrm{X}_{0}, 13 \mathrm{kGs}\right)$, is composed of two systems: the Liquid Xenon (LXe) calorimeter (about 5.4 $\mathrm{X}_{0}$ ), surrounded by the CsI crystal calorimeter [6] (about $8.1 \mathrm{X}_{0}$ ). The LXe calorimeter has 7 layers and utilizes dual readout: the anode signals are used for a measurement of the total energy deposition, while signals from the cathode strips provide information about a shower profile and are used to measure the coordinates of the photon conversion point to $1-2 \mathrm{~mm}$ precision. The endcap BGO crystal calorimeter (about $13.4 \mathrm{X}_{0}$ ) operates in the main magnetic field. The time-of-flight system, made of plastic scintillators with PMT readout, is placed between the two layers of the barrel calorimeter. The detector is surrounded by the muon range system.

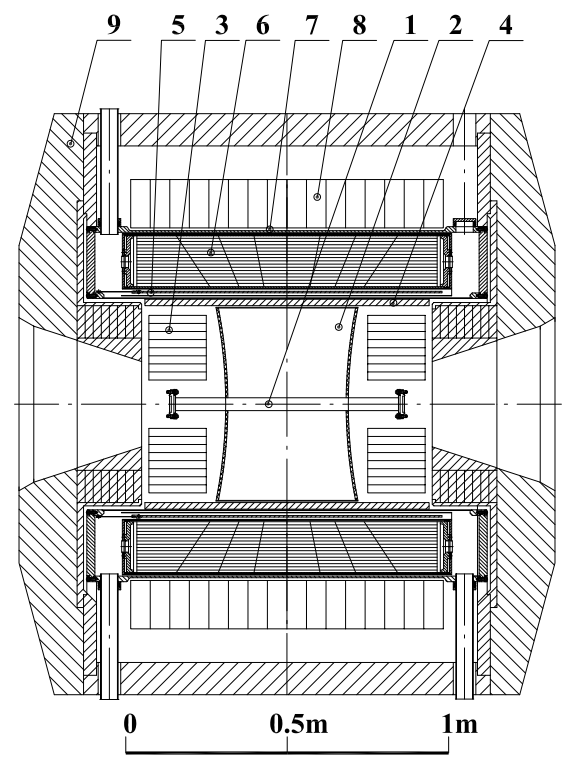

Figure 1. CMD-3 detector: 1 - beam pipe, 2 - drift chamber, 3 - BGO calorimeter, 4 - Z-chamber, 5 - superconducting solenoid, 6 - LXe calorimeter, 7 - TOF system, 8 - CsI calorimeter, 9 - yoke. The muon range system, not shown, is placed outside the yoke.

The primary goal of experiments at VEPP-2000 is a measurement of cross sections of various modes of $e^{+} e^{-} \rightarrow$ hadrons in the whole available c.m. energy range up to $2 \mathrm{GeV}$. The precision of the total cross section of $e^{+} e^{-} \rightarrow$ hadrons is closely related to the problem of the muon anomalous magnetic moment, $a_{\mu}$. The value of $a_{\mu}$ is predicted within the Standard Model (SM) to about $0.42 \mathrm{ppm}$. The accuracy of calculation is limited by the knowledge of the hadronic (QCD) contribution $a_{\mu}(\mathrm{had})$, which, in the lowest order, is calculated using dispersion relations by integrating $\sigma\left(e^{+} e^{-} \rightarrow\right.$ hadrons $)$. The dominant contribution to the integral comes from the low energy range accessible at VEPP-2000. Thus, VEPP-2000 measurements of $e^{+} e^{-} \rightarrow$ hadrons will help to reduce the uncertainty of the SM prediction for $a_{\mu}$. The most accurate measurement of $a_{\mu}$ was performed at BNL to $0.54 \mathrm{ppm}$ [7], and the result differs from the SM prediction by 3-4 standard deviations. A new experiment [8] aimed at measuring $a_{\mu}$ to $0.14 \mathrm{ppm}$ has collected first data in 2017. Another experiment is under construction at J-PARC [9]. 


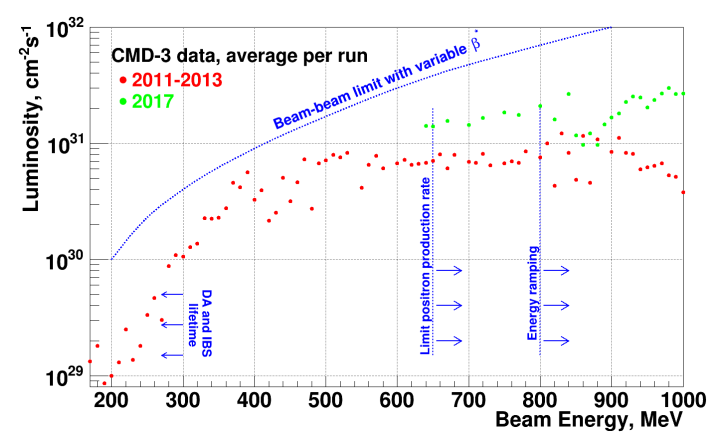

Figure 2. Average luminosity of VEPP-2000 at different energies. Red points — 2011-2013 runs, green points 2017 run.

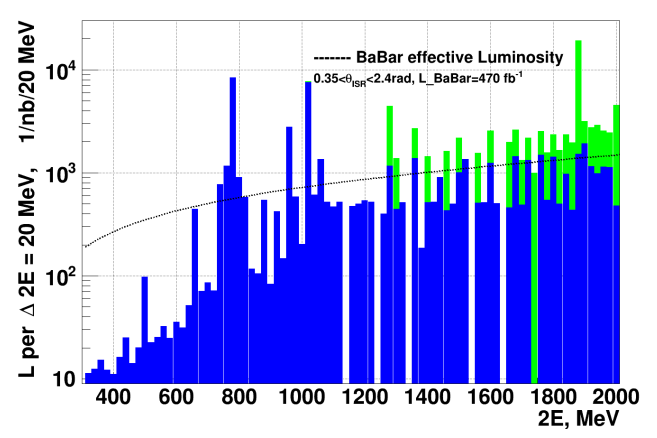

Figure 3. Integrated luminosity collected by CMD-3 at different energies with $20 \mathrm{MeV}$ energy bins. Blue - 2011-2013 runs, green - 2017 run. The line shows the effective ISR integrated luminosity at BaBar.

\section{Results from 2011-2013 runs}

The first round of data taking after VEPP-2000 commissioning was done in 2011-2013. In 2011 and 2012 the data were collected in the c.m. energy range from $1.0 \mathrm{GeV}$ to $2.0 \mathrm{GeV}$ with a $25 \mathrm{MeV}$ step in 2011 and slightly larger step in 2012. About $30 \mathrm{pb}^{-1}$ were collected, with about $10 \mathrm{pb}^{-1}$ above the $N \bar{N}$ threshold. In 2013 a c.m. energy scan from $1.0 \mathrm{GeV}$ down to $0.32 \mathrm{GeV}$ was performed in $20 \mathrm{MeV}$ steps, with finer steps up to $2 \mathrm{MeV}$ close to the $\omega(782)$ resonance. About $20 \mathrm{pb}^{-1}$ were collected, with about $8 \mathrm{pb}^{-1}$ near the $\omega(782)$ peak. In each season some amount of data was taken near the $\phi$ peak to calibrate the detector subsystems. Starting from 2012 the beam energy was continuously monitored with high precision $\Delta E / E<10^{-4}$ using Compton backscattering of laser photons at the electron beam $[10,11]$.

The collider performance is illustrated in Fig. 2. The peak VEPP-2000 luminosity observed in 2011-2013 was significantly lower than the design expectations at energies $\sqrt{s}>1.2 \mathrm{GeV}$, up to a factor of 10 at the maximum energy of $2 \mathrm{GeV}$. The main limitating factors were: the deficit of positrons significant for $\sqrt{s}>1.2 \mathrm{GeV}$, and the upper limit of the booster ring energy at 0.825 $\mathrm{GeV}$, which forced to ramp beam up and down at each injection into the main VEPP-2000 ring. The integrated luminosity collected in 2011-2013, is similar to that available at BaBar using ISR (Fig. 3).

The analysis of data collected in 2011-2013 is in progress. All major channels are under analysis including channels with up to 6 pions or 2 kaons and 2 pions in the final state. The luminosity is measured to $1 \%$ using $e^{+} e^{-} \rightarrow e^{+} e^{-}$and $e^{+} e^{-} \rightarrow \gamma \gamma$ events [12].

The CMD-3 collaboration published several results with a few charged particles in the final state: $e^{+} e^{-} \rightarrow 3\left(\pi^{+} \pi^{-}\right)$[13], $e^{+} e^{-} \rightarrow K^{+} K^{-} \pi^{+} \pi^{-}$[14], $e^{+} e^{-} \rightarrow p \bar{p}$ [15] and $e^{+} e^{-} \rightarrow K_{S} K_{L}$ [16] around the $\phi$-meson with $K_{S} \rightarrow \pi^{+} \pi^{-}$. The most recent results are the measurement of cross sections $e^{+} e^{-} \rightarrow$ $\pi^{+} \pi^{-} \pi^{0} \eta[17]$ and $e^{+} e^{-} \rightarrow \pi^{+} \pi^{-} \pi^{+} \pi^{-}$near $\phi(1020)$ [18].

The total cross section $e^{+} e^{-} \rightarrow \pi^{+} \pi^{-} \pi^{0} \eta$ was measured for the first time in the c.m. energy range from threshold to $2 \mathrm{GeV}$. Several intermediate states are identified: $\omega(782) \eta, \phi(1020) \eta, a_{0}(980) \rho(700)$ and an additional contribution possibly due to $\rho(1450,1700) \pi$.

The cross section $e^{+} e^{-} \rightarrow \pi^{+} \pi^{-} \pi^{+} \pi^{-}$was measured in the c.m. energy range between $0.922 \mathrm{GeV}$ and $1.06 \mathrm{GeV}$. Clear interference pattern between the non-resonant contribution and $\phi(1020) \rightarrow 4 \pi$ 


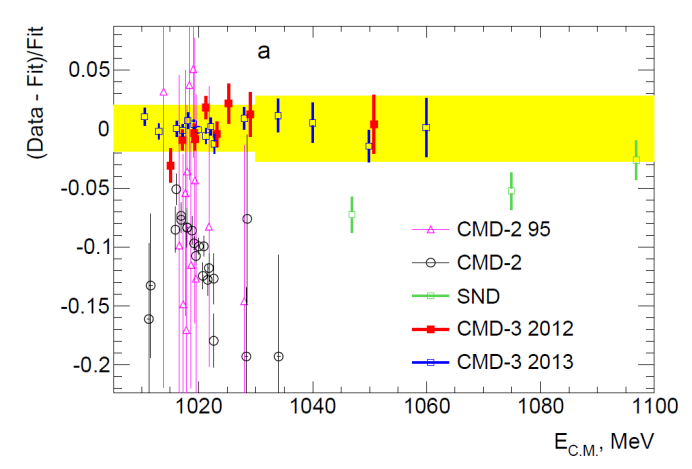

Figure 4. Comparison of the CMD-3 measurement of $e^{+} e^{-} \rightarrow K^{+} K^{-}$to previous measurements at CMD-2 $[19,20]$ and SND [21].

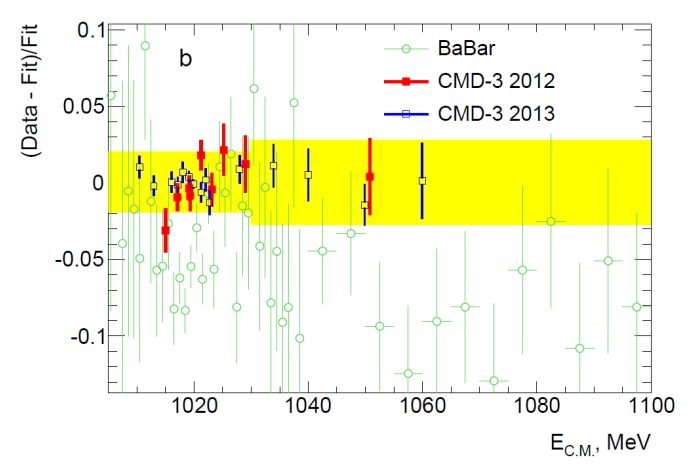

Figure 5. Comparison of the CMD-3 measurement of $e^{+} e^{-} \rightarrow K^{+} K^{-}$to the recent BaBar measurement [22] using ISR approach.

decay is observed, allowing to measure the branching ratio of G-parity and OZI-suppressed decay $B\left(\phi \rightarrow \pi^{+} \pi^{-} \pi^{+} \pi^{-}\right)=(6.5 \pm 2.7 \pm 1.6) \times 10^{-6}$.

The $e^{+} e^{-} \rightarrow K^{+} K^{-}$cross section was measured at the $\phi$-meson energy range to $2 \%$ systematic accuracy. The CMD-3 result is above all previous measurements [19-22] (Fig. 4 and 5) and is in perfect agreement with isospin symmetry: comparing with the recent CMD-3 measurement of $e^{+} e^{-} \rightarrow$ $K_{S} K_{L}$ [16], the ratio of coupling constants with the Coulomb factor taken into account

$$
\frac{g_{\phi K^{+} K^{-}}}{g_{\phi K_{S} K_{L}} \sqrt{Z\left(m_{\phi}^{2}\right)}}=0.990 \pm 0.017
$$

The preliminary results of the measurement of the dominant cross section $e^{+} e^{-} \rightarrow \pi^{+} \pi^{-}$are discussed in the separate paper [23].

\section{Collider and detector upgrades, 2013-2016}

After the first full scan of the whole VEPP-2000 energy range, the collider operation was suspended for 3 years to implement a number of improvements. A new injection complex VEPP-5 was commissioned at BINP in 2016. A new $200 \mathrm{~m}$ beamline was constructed, which connected the injection complex with VEPP-2000. That allowed to replace the old VEPP-2000 electron/positron source with the beams coming from the injection complex and completely resolved the problem of positron deficit. A second big improvement of VEPP-2000 was an increase of the maximum energy of the booster ring to match that of the main VEPP-2000 ring. That made it possible to inject beams to the main ring in the whole available energy range without energy ramping.

The major CMD-3 upgrade was a replacement of the time-of-flight system, located between the LXe and CsI barrel calorimeters [24, 25]. The new system is composed from about 200 strips of organic scintillator with shifter fiber readout and two silicon photomultiplier (SiPM) photodetectors per strip. The new system has high efficiency and time resolution of $0.5-0.7 \mathrm{~ns}$. Other detector upgrades include numerous improvements in electronics and DAQ hardware, including full replacement of electronics for readout of LXe calorimeter strips. 


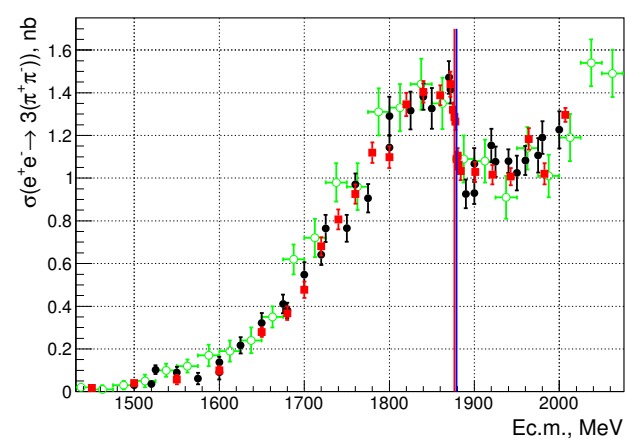

Figure 6. Preliminary results of the CMD-3 measurement of the $e^{+} e^{-} \rightarrow 3\left(\pi^{+} \pi^{-}\right)$cross section using 2017 data (red squares) in comparison with the previous CMD-3 measurement [13] (black dots) and BaBar measurement (green open circles).

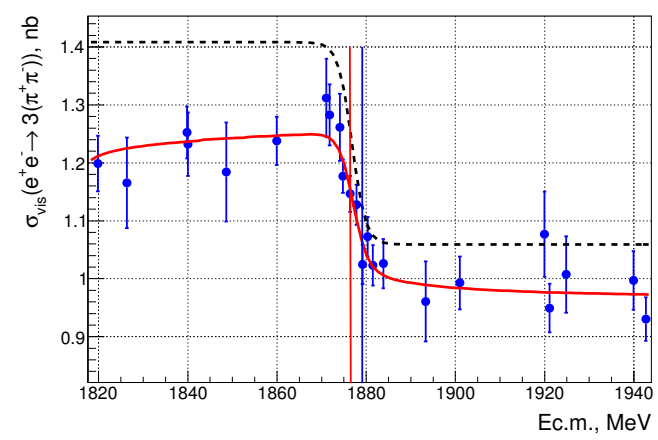

Figure 7. Fit of the $e^{+} e^{-} \rightarrow 3\left(\pi^{+} \pi^{-}\right)$cross section near the $N \bar{N}$ threshold (CMD-3, 2017 data). The curves are described in the text. The vertical lines show the $p \bar{p}$ and $n \bar{n}$ production thresholds.

The commissioning of the collider and the detector was done in 2016 and CMD-3 resumed data taking at the beginning of 2017. The VEPP-2000 luminosity in 2017 data taking is shown in Fig. 2 with green points. Significant improvement by a factor of 2 to 8 compared to 2011-2013 is observed.

\section{The 2017 run}

Overall, about $50 \mathrm{pb}^{-1}$ were collected in Feb.-Jun. 2017 in the energy range from $1.28 \mathrm{GeV}$ to 2.007 $\mathrm{GeV}$. The integrated luminosity collected at different energies is shown in Fig. 3 as a green histogram. About $30 \mathrm{pb}^{-1}$ were collected in $\approx 1 \mathrm{pb}^{-1}$ units distributed almost uniformly over the scanned energy range. The rest $20 \mathrm{pb}^{-1}$ were collected at two particular energies: $2.007 \mathrm{GeV}$ and near the $N \bar{N}$ threshold.

About $4 \mathrm{pb}^{-1}$ were collected at $\sqrt{s}=2.007 \mathrm{GeV}$, the highest energy accessible at VEPP-2000. These data were recorded to investigate a possibility of a search for direct production of $D^{* 0}(2007)$ in $e^{+} e^{-}$annihilation. Observation of such production at any level above the SM prediction $B\left(D^{* 0} \rightarrow\right.$ $\left.e^{+} e^{-}\right) \sim O\left(10^{-19}\right)$ would be a clear signal of physics beyond SM [26].

Previously, a drop of the cross section of $e^{+} e^{-} \rightarrow 3\left(\pi^{+} \pi^{-}\right)$at the $N \bar{N}$ threshold was observed [13]. To study the behaviour of various modes of $e^{+} e^{-} \rightarrow$ hadrons at the $N \bar{N}$ threshold, in 2017 we scanned the c.m. energy range around the threshold in small steps of $1.2 \mathrm{MeV}$, approximately equal to the beam energy spread. About $15 \mathrm{pb}^{-1}$ were collected in the energy range $1.872 \leq \sqrt{s} \leq 1.884 \mathrm{GeV}$.

The preliminary result of the measurement of the $e^{+} e^{-} \rightarrow 3\left(\pi^{+} \pi^{-}\right)$cross section using 2017 data is shown in Fig. 6. We confirmed the sharp drop in the cross section. The fit of the visible cross section with the step-like function

$$
\sigma_{\text {Born }}(E)=a+\frac{b}{1+\exp \left(\frac{E-E_{t h r}}{w}\right)},
$$

convolved with the beam energy spread and radiative correction, is shown in Fig. 7. The points are preliminary CMD-3 2017 data, the solid red curve is the fit of the visible cross section, and the black dotted curve is the corresponding $\sigma_{B o r n}(E)$. The obtained threshold energy $E_{t h r}=1876.81 \pm 0.07$ 
$\mathrm{MeV}$ is close to $2 m_{p}=1876.544 \mathrm{MeV}$. The width of the transition, corrected for the beam energy spread, is $w=1.496 \pm 0.063 \mathrm{MeV}$.

\section{References}

[1] P.Yu. Shatunov et al., Phys. Part. Nucl. Lett. 13 (2016) 995.

[2] D. Shwartz et al., PoS ICHEP2016 (2016) 054.

[3] B. I. Khazin et al., Nucl. Phys. Proc. Suppl. 181-182 (2008) 376.

[4] M. N. Achasov et al., Nucl. Instrum. Meth. A 598 (2009) 31.

[5] V. E. Shebalin et al., Nucl. Instrum. Meth. A 824 (2016) 710.

[6] V. M. Aulchenko et al., JINST 10 (2015) P10006.

[7] G. W. Bennett et al. [Muon g-2 Collaboration], Phys. Rev. D 73 (2006) 072003.

[8] I. Logashenko et al. [Muon g-2 Collaboration], J. Phys. Chem. Ref. Data 44 (2015) 031211.

[9] Naohito Saito [J-PARC g-'2/EDM Collaboration], AIP Conf.Proc. 1467 (2012) 45-56.

[10] E. V. Abakumova et al., Nucl. Instrum. Meth. A 744 (2014) 35.

[11] E. V. Abakumova et al., JINST 10 (2015) T09001.

[12] A. E. Ryzhenenkov et al., JINST 12 (2017) C07040.

[13] R. R. Akhmetshin et al., Phys. Lett. B 723 (2013) 82.

[14] D. N. Shemyakin et al., Phys. Lett. B 756 (2016) 153.

[15] R. R. Akhmetshin et al., Phys. Lett. B 759 (2016) 634.

[16] E. A. Kozyrev et al., Phys. Lett. B 760 (2016) 314.

[17] R. R. Akhmetshin et al., Phys. Lett. B 773 (2017) 150.

[18] R. R. Akhmetshin et al., Phys. Lett. B 768 (2017) 345.

[19] R. R. Akhmetshin et al., Phys. Lett. B 364 (1995) 199.

[20] R. R. Akhmetshin et al., Phys. Lett. B 669 (2008) 217.

[21] M. N. Achasov et al., Phys. Rev. D 94 (2016) 112006.

[22] J.P. Lees et al., Phys. Rev. D 88 (2013) 032013.

[23] F. Ignatov et al., "Preliminary results on CMD-3 measurement of $e^{+} e^{-} \rightarrow \pi^{+} \pi^{-}$cross section", this volume.

[24] G. V. Fedotovich et al., JINST 9 (2014) C09022.

[25] A. N. Kozyrev et al., JINST 12 (2017) C07027.

[26] A. Khodjamirian, T. Mannel, A. Petrov, JHEP 1511 (2015) 142. 